\title{
Estructura latente de una escala de anomia social e invarianza factorial en estudiantes peruanos de ambos sexos
}

\section{Latent structure of a social anomie scale and factor invariance in peru- vian students of both sexes}

\author{
Aaron Caycho Caja ${ }^{1}$ \\ Dirección Regional de Educación de Cajamarca
}

Recibido: $02-11$ - 20

Aceptado: 16 - $11-20$

Publicado: $21-12-20$

\section{Resumen}

El objetivo de la presente investigación fue hallar evidencia de la estructura latente de la Escala de Anomia social así como generar el apoyo empírico a la hipótesis de invarianza factorial según el sexo de 301 estudiantes preuniversitarios peruanos. Se realizó un análisis factorial confirmatorio de cada modelo, se halló la consistencia interna de la escala, y, luego, se planteó la invarianza factorial del Modelo 2020 propuesto. Los resultados señalan que el modelo de tres factores subyacentes no se sustenta en su consistencia interna; de manera alternativa, el modelo de dos factores consigue evidencia empírica a favor de su consistencia y estructura interna. Respecto a la invarianza factorial débil, esta no se justifica por problemas vinculados al grupo de mujeres. Se concluye que la Escala de Anomia Social evidencia dificultades asociadas a la presencia de la subescala de Pesimismo y en la evaluación de participantes mujeres.

Palabras clave: Invarianza factorial; consistencia interna; análisis factorial confirmatorio; anomia social.

\begin{abstract}
The objective of this research was to find evidence of the latent structure of the Social Anomie Scale as well as to generate empirical support for the factor invariance hypothesis according to the sex of 301 Peruvian pre-university students. A confirmatory factor analysis of each model was carried out, it was found support to the internal consistency of the scale, and then the factorial invariance of the proposed Model 2020 was raised. The results indicate that the model of three underlying factors is not sustained in its internal consistency; alternatively, the model of two factors obtains empirical evidence in favor of its consistency and internal structure. As for the weak invariance, this is not justified
\end{abstract}

1 Psicólogo de la Dirección Regional de Educación de Cajamarca.

E-mail: aaronkaycho@gmail.com ORCID: https://orcid.org/0000-0003-1478-8954

(C) Los autores. Este artículo es publicado por la Revista de Investigación en Psicología de la Facultad de Psicología, Universidad Nacional Mayor de San Marcos. Este es un artículo de acceso abierto, distribuido bajo los términos de la licencia Creative Commons Atribucion - No Comercia_Compartir Igual 4.0 Internacional. (http://creativecommons.org/licenses/by-nc-sa/4.0/) que permite el uso no comercial, distribución y reproducción en cualquier medio, siempre que la obra original sea debidamente citada. 
by problems linked to the group of women. It is concluded that the Social Anomie Scale shows difficulties associated with the presence of the subscale of Pessimism and in the evaluation of female participants.

Keywords: Factor invariance; internal consistency; confirmatory factor analysis; social anomie.

Anomia es un concepto con una larga tradición histórica que se remonta incluso a la edad antigua; sin embargo, su abordaje teórico es relativamente reciente. Durkheim (1893; 2012), en el anterior siglo, rescató el término para la sociología y menciona que esta se expresa en épocas de bruscas transiciones sociales en las cuales las personas experimentan que las normas tradicionales son imprecisas o poco claras debido a este cambio acelerado. Parsons (1966) señala que cuando las normas sociales aceptadas o institucionalizadas no son integradas, ocurre la anomia entendida como un quebrantamiento del orden social. Para Merton (1964), se pone en evidencia cuando los sujetos desean alcanzar los objetivos socialmente deseados, pero a través de medios reprobados.

El estudio a profundidad de la anomia llevó a su diferenciación conceptual desde la sociología y más orientada al empleo de indicadores socioeconómicos que hacen referencia a una anomia objetiva (Caycho, 2019a). Para la psicología, su estudio involucra una percepción de la ausencia de normas sociales, lo cual está enmarcado en la vertiente subjetiva de la anomia (Caycho, 2019a) que también guarda relación a las nociones tradicionales que brindan Merton (1964) o Durkheim (2012).

La diferenciación anterior ha permitido el posterior desarrollo de escalas psicométricas que midan la anomia psicológica (Srole, 1956). En el contexto latinoamericano, Aguiar (2003) realizó la validación de la escala de anomia social de Li, Atteslander, Tanur y Wang (1999) encontrando indicadores favorables de consistencia y estructura interna. Posteriormente, Formiga (2011), en una investigación realizada con estudiantes brasileños de ambos sexos encontró evidencia a favor de la estructura subyacente de esta versión $(\mathrm{GFI}=0.96, \mathrm{TLI}=1.00, \mathrm{CFI}=1.00$, RMSEA $=0.00$ ). En México, el estudio de Yañez (2011) permitió dar respaldo a la propuesta trifactorial de Aguiar. Respecto a este último, Yañez empleó como muestra a estudiantes de ambos sexos pertenecientes a escuelas públicas y utilizó un análisis factorial exploratorio (AFE) en el cual identificó tres dimensiones: Desconfianza, Descontento y Pesimismo, todas ellas con coeficientes alfa de consistencia interna de $.88, .80$ y .87 , respectivamente.

Además, diversas investigaciones psicométricas en Chile, Brasil y México han vinculado la anomia a otros conceptos como alienación (Aceituno, 1989; Vera, Yañez, Ramírez y Bautista, 2014), comportamiento desafiante (Bautista, 2014) o conducta antisocial y delictiva (Formiga, 2012). También se ha desarrollado modelos 
predictivos en los cuales la anomia participa como variable exógena explicativa de otros constructos como conducta antisocial (Formiga, 2012; Vera et al., 2014).

En el Perú, desde 1980, los artículos de la anomia han tomado en cuenta los postulados teóricos sociológicos para explicar fenómenos sociales como la migración interna del campo a la ciudad del siglo pasado y su impacto en la capital peruana (Lynch, 1989; Neira, 1987; Romero, 1987). Desde la psicometría, Caycho (2019b) realizó una validación de la Escala de Anomia Social de la versión mexicana (Yañez, 2011), la cual fue realizada en una población de pacientes drogodependientes. Se señala que el estudio de Caycho realizó un análisis factorial confirmatorio (AFC) por modelamiento de ecuaciones estructurales en el cual el ajuste del modelo replicó favorablemente la estructura interna de tres factores subyacentes al igual que reportó coeficientes de consistencia interna aceptables $(\omega>.70)$ en los tres constructos. No obstante, al ser una escala nueva, se encontraron limitaciones asociadas al tipo de muestra clínica empleada: drogodependientes varones en su mayoría jóvenes y adultos, lo cual limita su alcance a otras poblaciones.

En ese sentido, se busca replicar los hallazgos previos (Caycho, 2019b) respecto a la estructura y consistencia interna de la escala en muestra no clínica de estudiantes preuniversitarios de ambos sexos. Asimismo, el análisis de la invarianza factorial propuesto permite que la escala pueda ser utilizada de manera indistinta tanto en estudiantes varones como mujeres siempre y cuando los hallazgos respalden la hipótesis asociada a la invarianza de la medida entre ambos grupos.

\section{MÉTODO}

Se busca evidencia a favor de la estructura latente de tres factores de la Escala de Anomia Social a la vez que se procura coeficientes de consistencia interna favorables a la precisión de la medida de los constructos subyacentes, por lo cual se considera un estudio con un diseño instrumental (Ato, López y Benavente, 2013). A las mediciones señaladas se añade un aspecto medular básico asociado a la invarianza factorial entre los grupos de hombres y mujeres de la escala en cuestión.

\section{Participantes}

Se evaluaron 301 sujetos $(M=17.7, \mathrm{D} . \mathrm{S}=1.51)$, de los cuales 169 son de sexo masculino y 132 femenino. Los participantes son estudiantes preuniversitarios de dos academias de Lima ubicadas en el Cono Norte, los cuales cursan estudios con la intención de postular a carreras universitarias de diferentes centros de estudios de Lima.

\section{Instrumento}

Se empleó la escala de Inestabilidad Social (anomia social) elaborada por Li et al., (1999), adaptada en Brasil por Aguiar (2003) y, en México, por Yañez (2011). A 
partir de la adaptación de Yañez, en el Perú, Caycho (2019b) evaluó una muestra de pacientes drogodependientes varones de comunidades terapéuticas de Lima y replicó una estructura latente de tres factores subyacentes: Pesimismo, asociado a la percepción de oportunidades sociales futuras; Descontento, entendido como la comparación social que realiza un sujeto sobre la posición que ocupa en la sociedad; y Desconfianza, que evalúa el grado de credulidad que genera el Gobierno. El estudio de Caycho encontró coeficientes de bondad de ajuste adecuados (X2/ gl. $=1.46, \mathrm{SRMR}=0.068, \mathrm{RMSEA}=0.045, \mathrm{GFI}=0.970)$ al igual que coeficientes omega de consistencia interna entre moderados y satisfactorios en Pesimismo (0.71), Desconfianza (0.80) y Descontento (0.81), respectivamente. No obstante, se retiraron seis ítems con bajas saturaciones factoriales en la escala de Pesimismo; mientras que en la escala de Desconfianza únicamente un ítem. También se reportó la invarianza factorial entre los grupos de jóvenes y adultos, todos ellos de sexo masculino (Caycho, 2019b).

\section{Procedimiento}

Se pidió permisos al área de coordinación de las academias en mención procurándose la confidencialidad del nombre de los participantes los cuales firmaron un consentimiento informado. La aplicación del instrumento se realizó en dos oportunidades entre los meses de enero y febrero del 2020 y la evaluación tomó en cuenta los principios básicos de la Declaración de Helsinki respecto a los aspectos éticos en investigación.

\section{Análisis de datos}

Los análisis posteriores describen procedimientos estadísticos empleados para el tratamiento de los datos recolectados. Se señala que los hallazgos obtenidos fueron realizados a través del programa $\mathrm{R}$ studio, library Lavaan en la versión 4.0.3.

A nivel univariado se procuraron valores de kurtosis y asimetría en los límites sugeridos (+/- 1) a los cuales se les añade los índices estandarizados de asimetría (SSI) con valores entre los umbrales permisibles (SSI < 0.75) (Malgady, 2007). Se adiciona el análisis del efecto techo y piso (Terwee et al., 2007) de los puntajes situados en el extremo más alto o bajo de cada ítem que pueden representar una influencia negativa sobre la confiabilidad y la validez de contenido de la escala. Además, son tomados en cuenta los supuestos básicos del análisis multivariado: linealidad entre las variables, ausencia de multicolinealidad, tratamiento de datos atípicos y normalidad univariada y multivariada.

Con las consideraciones señaladas se realizó un Análisis Factorial Confirmatorio (AFC) con la estimación por Mínimos Cuadrados Ponderados Robustos (WLSMV) empleando la matriz de correlaciones policóricas acorde al tipo de escala Likert de la prueba. Para el presente, se realizó un AFC tomando en cuenta el análisis previo (Caycho, 2019b) el cual considera tres constructos subyacentes. No 
obstante, tuvo que realizarse un segundo modelo de AFC (Modelo 2020) con solo dos dimensiones en el cual no se incluyó la subescala de Pesimismo. Para el análisis de la bondad de ajuste se consideraron índices en los valores sugeridos: SRMR $\leq 0.08$, RMSEA $\leq 0.07, \mathrm{TLI} \geq 0.92, \mathrm{CFI} \geq 0.92$ (Hair, Black, Babin y Anderson, 2014) y un Chi cuadrado normado $1 \leq \mathrm{X} 2 / \mathrm{gl} \leq 5$ (Wu, 2013). También se reporta la consistencia de la escala a través del coeficiente omega $(\omega>0.70)$ y el coeficiente de Hancock $(\mathrm{H}>0.80)$ asociado a la confiabilidad del constructo, y se adicionan los valores del cálculo del monto promedio de la varianza extraída (AVE) que hace referencia a la validez convergente y discriminante.

Respecto a la invarianza factorial, esta se realizó poniendo a prueba el ajuste de la estructura latente de los modelos correspondientes a los grupos de hombres y mujeres. Asimismo, se plantearon de manera sucesiva contrastes entre los modelos de invarianza configural, débil, fuerte y estricta, de tal manera que la verificación de la hipótesis de invarianza de los valores obtenidos entre los grupos escogidos se afirme siempre y cuando se cumpla con la secuencia planteada y en los límites establecidos $(\triangle \mathrm{CFI} \leq 0.01$ y $\triangle \mathrm{RMSEA} \leq 0.015)$ para la comparación entre los modelos de invarianza mencionados.

\section{RESULTADOS}

El análisis descriptivo univariado (ver Tabla 1) de los datos señala linealidad entre las variables, ausencia de problemas de multicolinealidad. Asimismo, los valores de asimetría y kurtosis oscilan, en la mayoría de los ítems, en los valores establecidos $(+/-1)$, el SSI posee valores bajos $(<0.50)$ en casi todos los casos a excepción del ítem 6 y sí se observó el efecto techo o piso en una proporción mayor al 15\% en la mayoría de los ítems. Se señala que el empleo de la distancia de Mahalanobis evidenció la presencia de datos atípicos correspondientes a siete observaciones las cuales fueron retiradas por distanciarse del centroide multivariado. Además el test de Mardia no respalda la normalidad multivariante, pero a pesar de eso se puede proseguir con el AFC obviando este paso (Hair, Anderson, Tatham y Black, 1999).

Posteriormente, en vista de los estudios previos que refieren que la escala de Anomia Social está compuesta por tres dimensiones (Yañez, 2011), se planteó un AFC de la escala con la totalidad de los ítems de la misma manera que el estudio de Yañez; no obstante, se encontraron problemas de no convergencia, por lo cual se prosiguió con la propuesta del estudio peruano (Caycho, 2019b) en el cual se retiran los ítems 18,19,20,21,22 y 24 de la subdimensión Pesimismo, y el ítem 8 de Desconfianza. En este contexto, se realizó el AFC obteniéndose una mejoría en la estructura latente de la escala con índices de ajuste en los límites establecidos $(\mathrm{SRMR}=0.074, \mathrm{RMSEA}=.066$ [IC 90\% .055, .063], TLI $=0.949, \mathrm{CFI}=0.957 \mathrm{y}$ $\mathrm{x} / \mathrm{gl}=2.26$ ). Sin embargo, aparece un problema asociado a la consistencia interna en la subdimensión de Pesimismo en la cual el coeficiente omega (0.60) y la H de Hancock (0.69) se encuentran por debajo de los límites establecidos. Estos valores 
en parte evidencian una inquietud del estudio previo (Caycho, 2019b) en el cual la subdimensión de Pesimismo ya mostraba ítems problemáticos y baja consistencia interna $(\omega=0.71)$. Adicionalmente, se hallaron cargas factoriales bajas $(\lambda<0.45)$ en el ítem 13 de la escala problema de Pesimismo y el ítem 16 de Desconfianza. Adicionalmente el valor del AVE de las dimensiones brinda respaldo a la validez interna discriminante de las subescalas (AVE $>\mathrm{R}^{2}$ ), pero no apoya a la validez convergente $(\mathrm{AVE}<0.5)$.

\section{Tabla 1}

Estadisticos descriptivos de la Escala de Anomia Social

\begin{tabular}{|c|c|c|c|c|c|c|c|}
\hline Ítem & M & D.E & g1 & g2 & Mín. \% & Máx. \% & SSI \\
\hline 1 & 2.5 & 1.02 & 0.33 & -0.45 & 16.8 & 3.1 & 0.16 \\
\hline 2 & 2.68 & 0.94 & 0.2 & -0.25 & 9.6 & 3.1 & 0.11 \\
\hline 3 & 2.33 & 1.07 & 0.68 & -0.12 & 22.6 & 4.5 & 0.30 \\
\hline 4 & 2.21 & 1.07 & 0.62 & -0.26 & 31.5 & 3.4 & 0.27 \\
\hline 5 & 3.68 & 1.25 & -0.69 & -0.61 & 7.5 & 31.8 & -0.22 \\
\hline 6 & 1.89 & 1.11 & 1.31 & 1.03 & 48.3 & 5.1 & 0.53 \\
\hline 7 & 2.02 & 1.14 & 1.06 & 0.29 & 41.8 & 4.8 & 0.41 \\
\hline 8 & 2.73 & 1.36 & 0.26 & -1.21 & 22.9 & 13 & 0.07 \\
\hline 9 & 3.92 & 1.08 & -0.63 & -0.72 & 1 & 38.7 & -0.27 \\
\hline 10 & 3.59 & 1.06 & -0.34 & -0.55 & 3.1 & 22.9 & -0.15 \\
\hline 11 & 2.39 & 0.94 & 0.44 & 0.08 & 17.5 & 2.7 & 0.25 \\
\hline 12 & 2.09 & 0.98 & 0.82 & 0.47 & 30.5 & 2.7 & 0.43 \\
\hline 13 & 3.08 & 1.08 & 0.06 & -0.51 & 7.2 & 11.6 & 0.03 \\
\hline 14 & 3.44 & 1.12 & -0.26 & -0.93 & 3.4 & 19.2 & -0.10 \\
\hline 15 & 3.18 & 1.13 & -0.01 & -0.91 & 5.5 & 13.7 & 0.00 \\
\hline 16 & 3.16 & 1.11 & -0.09 & -0.79 & 6.5 & 12 & -0.04 \\
\hline 17 & 2.48 & 1.05 & 0.63 & 0.03 & 16.1 & 6.2 & 0.29 \\
\hline 18 & 4.6 & 0.83 & -2.2 & 4.35 & 1 & 76.7 & -1.60 \\
\hline 19 & 3.08 & 1.06 & 0.01 & -0.3 & 8.2 & 11.6 & 0.00 \\
\hline 20 & 2.47 & 1.35 & 0.56 & -0.87 & 30.8 & 12 & 0.15 \\
\hline 21 & 3.32 & 1.27 & -0.17 & -1.02 & 8.9 & 24 & -0.05 \\
\hline 22 & 2.53 & 1.09 & 0.47 & -0.52 & 16.1 & 4.8 & 0.20 \\
\hline 23 & 3.45 & 1 & -0.36 & -0.27 & 3.8 & 14 & -0.18 \\
\hline 24 & 2.76 & 1.12 & 0.37 & -0.55 & 11.6 & 8.9 & 0.15 \\
\hline
\end{tabular}

Nota: g1, asimetría de Fisher; g2, kurtosis de Fisher; M, media; D.S, desviación estándar; SSI, índice de asimetría; Mín. \%, porcentaje de personas con puntaje mínimo; Máx. \%, porcentaje de puntaje máximo; en negrita, valores fuera de los rangos establecidos.

Por otro lado, como parte del estudio es probar la pertinencia de la escala tanto en varones y mujeres, se realizó dos AFC tanto en el grupo de hombres $(\mathrm{N}=169)$ y mujeres (132) encontrándose los mismos problemas de baja consistencia en ambos grupos.

Bajo estas condiciones, se planteó retirar la subescala problemática de Pesimismo y realizar un AFC con las escalas restantes. En tal sentido, se consideró emplear las subdimensiones de Desconfianza y Descontento de la versión inicial de Yañez 
(2011), manteniendo solo los ítems con saturación factorial favorable $(\lambda>0.45)$, por lo cual se retiró el ítem 16 del estudio de Caycho (2019b). Este modelo de dos factores (Modelo 2020) mejora en bondad de ajuste $(\mathrm{SRMR}=0.063, \mathrm{RMSEA}=0.056[\mathrm{IC}$ $90 \% .055, .056], \mathrm{TLI}=0.977, \mathrm{CFI}=0.982 \mathrm{y} \mathrm{x} / \mathrm{gl}=1.91)$ y las subescalas de Descontento y Desconfianza mantienen índices de consistencia elevados $(\omega \geq 0.79, \mathrm{H}>.80)$. Además, las cargas factoriales son de moderadas a altas $(\lambda>0.45)$ y el AVE apoya a la diferenciación entre ambas subescalas $\left(\mathrm{AVE}>\mathrm{R}^{2}\right)$ (ver Tabla 2).

\section{Tabla 2}

Cargas factoriales, consistencia interna y AVE de la Escala de Anomia Social del modelo de Caycho (2019b) y de la versión propuesta en el Modelo 2020

\begin{tabular}{|c|c|c|c|c|c|}
\hline \multicolumn{6}{|c|}{ Caycho 2019b } \\
\hline ítem & F1 & Ítem & F2 & ítem & F3 \\
\hline 5 & 0.587 & 1 & 0.699 & 4 & 0.543 \\
\hline 9 & 0.562 & 2 & 0.640 & 6 & 0.757 \\
\hline 10 & 0.687 & 3 & 0.840 & 7 & 0.524 \\
\hline 14 & 0.632 & 11 & 0.760 & 13 & 0.212 \\
\hline 15 & 0.642 & 12 & 0.641 & & \\
\hline 16 & 0.440 & 17 & 0.564 & & \\
\hline 23 & 0.556 & & & & \\
\hline$\omega$ & 0.787 & & 0.848 & & 0.596 \\
\hline $\mathrm{H}$ & 0.797 & & 0.868 & & 0.686 \\
\hline $\mathrm{F} 1$ & 1 & & 0.29 & & -0.18 \\
\hline $\mathrm{F} 2$ & $* 0.08$ & & 1 & & -0.17 \\
\hline F3 & $* 0.03$ & & $* 0.03$ & & 1 \\
\hline AVE & 0.35 & & 0.49 & & 0.30 \\
\hline \multicolumn{4}{|c|}{ Modelo 2020} & & \\
\hline ítem & F1 & ítem & F2 & & \\
\hline 5 & 0.605 & 1 & 0.699 & & \\
\hline 8 & 0.481 & 2 & 0.638 & & \\
\hline 9 & 0.575 & 3 & 0.837 & & \\
\hline 10 & 0.682 & 11 & 0.766 & & \\
\hline 14 & 0.624 & 12 & 0.639 & & \\
\hline 15 & 0.614 & 17 & 0.564 & & \\
\hline 16 & retirado & & - & & \\
\hline 23 & 0.568 & & - & & \\
\hline $\mathrm{R}$ & 0.291 & $\mathrm{R}^{2}$ & $* 0.08$ & & \\
\hline$\omega$ & 0.792 & & 0.847 & & \\
\hline $\mathrm{H}$ & 0.798 & & 0.868 & & \\
\hline AVE & 0.32 & & 0.48 & & \\
\hline
\end{tabular}

Nota: F1, Desconfianza; F2, Descontento; F3, Pesimismo; $(*)$, correlación al cuadrado entre los factores; R, correlación. 


\section{Invarianza factorial}

El análisis de la invarianza factorial implica que al ser evaluado un grupo heterogéneo de una población, la medición de sus propiedades debe ser equivalente entre sus subgrupos (Brown, 2015). En el presente, se asume que el Modelo 2020 propuesto debe ser idéntico en ambos sexos. En ese sentido, se muestra la bondad de ajuste del AFC que se realiza en el grupo de hombres y mujeres, encontrándose que este último muestra índices de ajuste fuera de los umbrales establecidos, lo cual ya hace referencia a dificultades estructurales de la escala. Luego, cuando se obtiene el modelo configural, los índices de ajuste se encuentran al borde de los límites establecidos, pero los índices del modelo de invarianza débil ya caen fuera de este rango, lo cual ya evidencia las dificultades asociados a ambos grupos e implica detener posteriores análisis ya que no se cuenta con el respaldo estadístico suficiente. Además, de continuar con el análisis se observa que la invarianza fuerte tampoco se sostendría debido a la diferencia entre su ajuste y la invarianza débil $(\triangle \mathrm{RMSEA}=.016)($ ver Tabla 3$)$.

Adicionalmente, con el objetivo de analizar los hallazgos concernientes a la invarianza factorial, fue calculada la media de las puntuaciones de la escala de anomia social tanto en varones $(\mathrm{M}=2.76)$ como en mujeres $(\mathrm{M}=2.62)$, y al compararse ambas mediante una prueba $t$ de student no se encontraron diferencias estadísticamente significativas entre estas, lo cual si bien no permite afirmaciones categóricas, apertura diferentes posibilidades a tomar en cuenta en la discusión de los resultados.

Tabla 3

Invarianza factorial de la Escala de Anomia Social en estudiantes varones y mujeres

\begin{tabular}{|c|c|c|c|c|c|c|}
\hline Modelo & $X^{2} / g . l$ & CFI & $\Delta$ CFI & RMSEA [IC90\%] & ARMSEA & SRMR \\
\hline hombre & 1.36 & .987 & & $.048(.016-.071)$ & & .073 \\
\hline mujeres & 2.01 & .964 & & $.088(.066-.109)$ & & .091 \\
\hline configural & 1.69 & .975 & & $.069(.053-.084)$ & & .081 \\
\hline Débil & 1.76 & .970 & .005 & $.072(.057-.087)$ & .003 & .086 \\
\hline Fuerte & 2.02 & .977 & .007 & $.056(.040-.070)$ & .016 & .084 \\
\hline estricta & 1.47 & .975 & .002 & $.057(.042-.071)$ & .001 & .086 \\
\hline
\end{tabular}

\section{DISCUSIÓN}

El estudio de la estructura latente e invarianza factorial de la Escala de Anomia Social devela puntos inquietantes no considerados en los estudios previos (Caycho, 2019b; Yañez, 2011) que a continuación se analizan.

Se puso a prueba un primer modelo considerando el estudio inicial de México (Yañez, 2011), pero este tuvo problemas de no convergencia lo cual ya alertaba de 
las dificultades posteriormente halladas. No obstante, si bien el modelo planteado de acuerdo a la versión peruana (Caycho, 2019b) mejoró, no puede ser considerado adecuado debido a los problemas presentados en la subescala de Pesimismo. Esto ya se visualizaba de alguna manera en la escala peruana anterior en la cual se eliminaron los ítems 18, 19, 20, 21, 22 y 24 de Pesimismo, pero se mantuvieron ítems con saturaciones factoriales mayores a 0.44 en los restantes ( ítems 4, 6, 7 y 13). En el presente análisis los ítems 4,6 y 7 indican saturaciones mayores a 0.50 a excepción del ítem 13 "la inflación está en aumento descontroladamente" $(\lambda=.21)$. Probablemente la baja varianza común del ítem 13 tenga que ver con que este no está claramente asociado a los ítems 4 "En términos generales, la distancia entre ricos y pobres está aumentando injustamente", 6 "La corrupción política actual se está transformando en una moda social", y 7 "En nuestro país no existe un sistema social justo o esa cosa de derechos iguales para todos" que implican cómo el sujeto valora las oportunidades sociales accesibles a este. Si bien en el Perú la inflación era un tema común y angustiante en el periodo de gobierno entre 1980 y 1990, en la actualidad no reviste un tema que genere preocupación en un nivel superlativo o que implique la atención angustiante del ciudadano; de ahí que este ítem no logre identificarse propiamente con esta subescala. Sin embargo, en la versión de Caycho (2019b) este ítem satura con una carga factorial más alta $(\lambda=0.54)$ que el presente $(\lambda=0.21)$ lo cual puede explicarse por la media de edad del primero $(\mathrm{M}=$ 28.3) más alta que el aquí evaluado $(\mathrm{M}=17.7)$, lo cual lo hace menos sensible a las respuestas de los estudiantes que incluso no habían nacido en esa época.

Respecto a los ítems 4, 6 y 7, el análisis descriptivo revelan la presencia del efecto piso en ambos así como también valores SSI de 0.53 en el ítem 6. Estos resultados posiblemente tengan que ver con las dificultades observadas en la precisión de la medición reflejada en la baja consistencia interna de la escala ya que, en el caso de estos ítems, los sujetos con los puntajes más bajos no podrían diferenciarse entre ellos (Terwee et al., 2007). Asimismo, un análisis del contenido teórico de las escalas refleja tanto en Desconfianza y Descontento homogeneidad entre sus ítems ya que en el caso de Descontento todos sus ítems hacen referencia al grado de satisfacción social conseguido y en Descontento, la confianza depositada en el gobierno. En el caso de Pesimismo, los ítems, 4, 6 y 7 hacen alusión a un tipo de contenido asociado a la justicia social que al parecer no estaría propiamente orientado a las posibilidades de oportunidades futuras al cual hace referencia el constructo, además de mostrar cierta heterogeneidad entre ellos. Probablemente, la media de edad ( $M=28.3)$ del estudio de Caycho (2019b) influye en el funcionamiento de estos ítems más susceptibles a las personas que han vivido periodos en el cual la injusticia social ha sido más determinante considerando sobre todo que pasada la década del 1980 - 1990, el Perú creció en términos económicos (Chirinos, 2008), por lo cual estos ítems no son tan sensibles en la generaciones posteriores tal y como la población de este estudio $(\mathrm{M}=17.7)$. Adicionalmente, el ítem 7 incluye una partícula negativa "En nuestro país no existe un sistema social 
justo o esa cosa de derechos iguales para todos", la cual es poco aconsejado en la formulación de ítems. Se añade que si existe la intención de mantener esta subescala, podría considerarse el reformular los ítems de tal manera que puedan reflejar de manera fidedigna al constructo de Pesimismo.

Además, a pesar de contar con una bondad de ajuste moderadamente aceptable, el modelo de Caycho (2019b) no se respalda por la baja consistencia interna de la subescala de Pesimismo $(\omega=.60, \mathrm{H}=.69)$. Se señala que en el estudio de Yañez (2011) la subescala obtenía un coeficiente de alfa mayor a 0.80; no obstante, este coeficiente es más sensible a la cantidad de ítems a comparación del coeficiente omega empleado, cuyo valor representa una medida más adecuada de la consistencia interna de la presente escala. A partir de estas dificultades es que pasa a cuestionarse la pertinencia de la subescala de Pesimismo y sus ítems, motivo por el cual fue posteriormente retirada.

Luego de la evaluación del modelo de Caycho (2019b), se postuló el Modelo 2020 omitiendo la escala problemática de Pesimismo, consiguiéndose una mejora sustancial en la estructura latente de dos factores, los cuales poseen saturaciones factoriales moderadas $(\lambda>0.5)$ en sus dos dimensiones. Asimismo, los coeficientes de consistencia de Desconfianza $(\omega=0.79, \mathrm{H}=0.80)$ y Descontento $(\omega=0.85$, $\mathrm{H}=0.85)$ muestran valores adecuados y los valores del AVE respaldan la validez interna discriminante entre las dos subescalas. Como se mencionó, ambas escalas son las que se han mantenido estables en el estudio previo (Caycho, 2019b) y el actual, mostrando en su conjunto una adecuada estructura factorial y consistencia interna, la cual también fue evidenciada en el estudio mexicano (Yañez, 2011).

De acuerdo al modelo propuesto se pasó a realizar la medición de la invarianza factorial, la cual es necesaria para determinar la pertinencia de las evaluaciones con grupos diversos, pero que no ha sido considerada en los estudios de anomia social realizados en Latinoamérica (Aceituno et al., 2009; Formiga 2011; Yañez, 2011). Sobre el estudio realizado por Caycho (2019b), este empleó una medida de la invarianza factorial considerando los grupos de jóvenes y adultos, ambos varones, los cuales se muestran invariantes; no obstante, la muestra estaba constituido por varones drogodependientes con una media de edad de 28.3 años, características importantes a considerar ya que pueden explicar muchos de los resultados obtenidos.

Merton (1964) señala que un modo de adaptación a los periodos críticos es el retraimiento o alejamiento de las metas o aspiraciones sociales, aspecto similar al presentado en muchos alcohólicos o drogodependientes. Al parecer la subescala de Pesimismo y las características del estudio de Caycho (2019b) serían más adecuadas para evaluar un tipo de muestra con características más afines, incluso a nivel teórico, a las conductas antisociales presentes en la muestra drogodependiente. Se señala que los estudios de anomia social han sido frecuentemente asociados 
a la conducta antisocial y delictiva (Formiga, 2011; Formiga, 2012; Vera, Bautista, Ramírez y Yañez, 2012; Vera et al., 2014), pero no se ha considerado las diferencias del sexo entre los participantes, lo cual habría hecho más sensible la escala de Caycho (2019b) a la muestra de varones. Además, la muestra presente corresponde a estudiantes de academias preuniversitarias, quienes se caracterizan por poseer intereses prosociales vinculados a ejercer una carrera profesional. Este grupo es cualitativamente diferente de la muestra clínica de drogodependientes, más propenso a la conducta antisocial, o de grupos de estudiantes de educación secundaria, probablemente con otras características psicológicas.

Asimismo, al analizar los valores del AFC entre hombres y mujeres por separado, considerados en la invarianza factorial, se observa cómo la escala muestra excelente bondad de ajuste y consistencia interna para el grupo de varones, pero el ajuste es pésimo para el caso de las mujeres. Además, al hacer una comparación entre la media de las puntuaciones del grupo de hombres y el de mujeres, se halló al primero con un valor ligeramente mayor, aunque estadísticamente no significativo. Se podría señalar al sexo como una característica que guarda mayor relación a la conducta antisocial, (uso de drogas, por ejemplo) más frecuente en varones ya sea en los grupos invariantes de jóvenes o adultos (Caycho, 2019b). En el presente caso, la negativa al apoyo de la bondad de ajuste del AFC en mujeres muestra cómo la escala no realiza una medición precisa de la anomia social en el grupo de mujeres, lo cual se refleja en el rechazo a la invarianza débil del estudio.

Como se mencionó, los estudios previos no han considerado este aspecto relevante el cual al parecer, puede estar ocultando un aspecto importante a considerar desde la teoría de la anomia, la cual se ha asociado a la conducta del delito o la criminalidad. Respecto a la población femenina, llama la atención que ítems orientados a medir la confianza en el gobierno o la satisfacción social conseguida no reflejen sus valoraciones tal como se hubiese esperado. Solamente este elemento puede involucrar muchas aristas, pero del cual solo se podría decir que guarda relación con la situación de exclusión social, la cual podría estar siendo percibida aún de manera benévola por este propio grupo o quizás este institucionalizada (Parsons, 1966).

Por otra parte, como limitaciones del estudio se señalan la muestra no probabilística de los participantes y la ausencia de variables socioeconómicas o de otros grupos etarios a considerar entre hombres y mujeres. Además, el recojo de los datos se realizó en el primer trimestre del año, lo cual reflejaría un menor grado de anomia y mostraría una imagen un tanto favorable y positiva de la sociedad a comparación de los cambios posteriores provocados por la pandemia y más acorde a los procesos críticos vinculados a la anomia social.

Finalmente, los hallazgos obtenidos señalan problemas métricos en las escala de Pesimismo. En cuanto a las subescalas de Descontento y Desconfianza 
del Modelo 2020 propuesto, ambas reflejan características más precisas en consistencia interna y estructura latente en los estudiantes preuniversitarios varones pero no en el grupo de mujeres; este último probablemente evidencie aspectos sociopsicosociales particulares que aún deben investigarse a detalle. En el presente caso, la invarianza factorial es una condición importante a evaluar sobre todo cuando se toma en cuenta el sexo de los participantes. No obstante, en vista de ser una escala nueva en el Perú se sugiere mayores estudios que ahonden en las características psicométricas de la prueba incluso considerando la subescala de Pesimismo; más aún si se considera que esta y el total de la escala pueden ser susceptibles a las brechas sociales que se avizoran en el país peruano, las mismas que se han exacerbado como producto de la pandemia mundial del presente año 2020. Adicionalmente, es pertinente una revisión teórica de la anomia y la forma en cómo se manifiesta en el grupo de mujeres, lo cual podría derivar en el replanteamiento de algunos ítems o la inclusión de otros más sensibles a esta muestra.

\section{CONCLUSIONES}

- El modelo de tres factores subyacentes de Caycho (2019b) no posee apoyo empírico por dificultades evidenciadas en la subescala de Pesimismo.

- La propuesta del Modelo 2020 de la Escala de anomia social evidencia estructura y consistencia interna favorable cuando no se considera la distinción de grupos según el sexo.

- La hipótesis de invarianza factorial del Modelo 2020 según el sexo no se mantiene debido a problemas en la estructura latente del grupo de mujeres.

\section{Fuentes de financiamiento}

El presente estudio fue autofinanciado.

\section{Aspectos éticos}

El proyecto de investigación fue presentado a la coordinación académica de las dos academias donde se realizó y contó con la aprobación del coordinador principal de cada una de ellas. Asimismo, los participantes dieron el consentimiento al estudio, el cual fue realizado en condiciones de anonimato y de acuerdo a los principios señalados en la Declaración de Helsinki de 1975.

\section{Conflicto de intereses}

El autor declara no tener ningún tipo de conflicto de intereses. 


\section{REFERENCIAS}

Aceituno, R. (1989). Anomia y alienación en estudiantes de cuarto medio de Santiago. Un estudio exploratorio. Memoria no publicada para optar al título de Psicólogo, Departamento de Psicología, Facultad de Ciencias Sociales, Universidad de Chile. Santiago, Chile.

Aceituno, R., Asún, R., Ruiz, S., Reinoso, A., Venegas, J.I., y Corbalan, F. (2009). Anomia y alienación en estudiantes secundarios de Santiago de Chile: Resultados iniciales de un estudio comparativo 1989-2007. Psykhe, 18 (2), 3-18. doi: 10.4067/S071822282009000200001

Aguiar, M. (2003). Representação social da sociedade, anomia e individualismo-coletivismo. Dissertação de mestrado não publicada. Universidade Federal do Rio de Janeiro. Rio de Janeiro, Brasil.

Ato, M., López, J., y Benavente, A. (2013). Un sistema de clasificación de los diseños de investigación en psicología. Anales en psicología, 29(3), 1038-1059. doi: 10.6018/ analesps.29.3.178511

Bautista, G. (2014). Conducta antisocial, anomia y alienación en adolescentes con diferentes niveles de socialización en Hermosillo. (Tesis de maestría). Centro de Investigación en alimentación y Desarrollo, A.C. Sonora, México.

Caycho, A. (2019). Sociopsicología de la anomia. Revista de investigación en psicología, 21(2), 285-300. doi:10.15381/rinvp.v21i2.15828

Caycho, A. (2019). Propiedades psicométricas de la Escala de Anomia Social en pacientes drogodependientes peruanos. Interacciones, 5(1), 17-23. doi: 10.24016/2019.v5n1.164

Chirinos, R. (2008). ¿Puede el Perú ser un nuevo milagro económico? Lima, Perú: Banco Central de Reserva del Perú. Recuperado de http://www.bcrp.gob.pe/

Durkheim, E. (2012). La división del trabajo social. Madrid, España: Biblioteca Nueva.

Formiga, N., y Fernandes, C. I. (2011). Um nexo causal entre variáveis da violencia em jovens. Cad. de Pesq. Interdisc. em Ci-s. Hum-s., 12 (100), 86-104. Recuperado de:https://periodicos.ufsc.br/index.php/cadernosdepesquisa/article/viewFile/19848951.2011v12n100p86/18557

Formiga, N. (2012a). Verificacao de um modelo causal entre anomia social e condutas desviantes em jovens. Encontro, Revista de psicologia, 15 (22), 77 - 94. Recuperado de: http://revista.pgsskroton.com.br/index.php/renc/article/download/2482/2377

Hair, J., Anderson, R., Tatham, R., y Black, W. (1999). Análisis multivariante. 5a Ed. Madrid, España: Prentice Hall.

Hair, J., Black, W., Babin, B., y Anderson, R. (2014). Multivariate data analysis. Seventh Edition. Edinburgh Gate, United Kingdom: Pearson.

Li, H., Atteslander, P., Tanur, J., y Wang, Q. (1999). Anomie scales: measuring social instability. En A. Peter, B. Gransow y J. Western (Eds.). Comparative anomie research: hidden barriers-hidden potential for social development). Sidney, Australia: Ashgate. 
Lynch, N. (1989). "Anomia de Regresión o Anomia de Desarrollo?". Socialismo y participación, 45, 39 - 52. Recuperado de: http://www.acuedi.org/ddata/65.pdf

Malgady, R. (2007). How skewed are psychological data? A standardized index of effect size. The Journal of General Psychology, 134(3), 355-359. doi:10.3200/ GENP.134.3.355-360

Merton, R. (1964). Teoría y estructuras sociales. México DF: Fondo de Cultura Económica.

Neira, H. (1987). Violencia y anomia: reflexiones para comprender. Socialismo y participación, 37, 1 -13. Recuperado de: http:/www.bloghugoneira.com/wp-content/ uploads/2012/02/ViolenciayAnomia1987.pdf

Parsons, T. (1966). El sistema social. Madrid, España: Biblioteca de la Revista de Occidente.

Romero, C. (1987). "Violencia y anomia: comentarios sobre una reflexión". Socialismo Participación, 39, 75 - 80.

Srole, L. (1956). Social integration and certain corollaries: An exploratory study. American Sociological Review, 21(6), 709-716. doi: 10.2307/2088422

Terwee, C. B., Bot, S. D., Boer, M. R. van der Windt, D. A., Knol, D. L., Dekker, J.,Bouter, L. M., y de Vet, H. C. (2007). Quality criteria were proposed for measurement properties of health status questionnaires. Journal of Clinical Epidemiology, 60(1), 3442. doi:10.1016/j.jclinepi.2006.03.012

Vera, J., Bautista, G., Ramírez, M., y Yañez, A. I. (2012). Relación entre anomia social, alienación y conducta antisocial en jóvenes infractores mexicanos. Revista Latinoamericana de Ciencias Sociales, Niñez y Juventud, 10(2), 943-955. Recuperado de: https://www.redalyc.org/html/773/77323978012/

Vera, J. A., Yáñez, A. I., Ramírez, M., y Bautista, G. (2014). Anomia social, anomia psicológica y alienación como predictores de la conducta disocial en Adolescentes escolares. Pesquisas e Práticas Psicossociais, 8 (2), 226-233. Recuperado de: http://www. seer.ufsj.edu.br/index.php/revista_ppp/article/view/535

Wu M. L. (2013). Questionnaire Statistical Analysis Practice-SPSS Operation and Application. Chongqing: Chongqing University Press.

Yáñez, A. (2011). Propiedades psicométricas de las escalas de Anomia y Desarrollo Moral en una muestra de Estudiantes de Bachillerato. Tesis publicada para obtener el título de licenciado en psicología. Universidad de Sonora, Sonora, México. 